\title{
Apoptin Mediates Endogenous Mitophagy and Apoptosis by Regulating the Level of ROS in Hepatocellular Carcinoma
}

Yiquan Li ( $005381255 @ q q . c o m$ )

Changchun University of Chinese Medicine https://orcid.org/0000-0002-6795-6088

Chao Shang

Changchun University of Chinese Medicine

Zirui Liu

Changchun University of Chinese Medicine

Jicheng Han

Changchun University of Chinese Medicine

Wenjie Li

Wenzhou University

Pengpeng Xiao

Wenzhou University

Nan Li

Wenzhou University

Shanzhi Li

Changchun University of Chinese Medicine

Zhiru Xiu

Changchun University of Chinese Medicine

Gaojie Song

Changchun University of Chinese Medicine

Yaru Li

Changchun University of Chinese Medicine

Ningyi Jin

Changchun University of Chinese Medicine

Jinbo Fang

Changchun University of Chinese Medicine

Xiao Li

Changchun University of Chinese Medicine

Yilong Zhu

Changchun University of Chinese Medicine 
Research

Keywords: Apoptin, human liver cancer, apoptosis, autophagy, ROS

Posted Date: December 29th, 2021

DOI: https://doi.org/10.21203/rs.3.rs-1163939/v1

License: (c) (i) This work is licensed under a Creative Commons Attribution 4.0 International License. Read Full License 


\section{Abstract}

Background: Apoptin, as a tumor-specific pro-apoptotic protein, apoptin plays an important role in the field of anti-tumor, but its autophagy activation mechanism and the interaction between autophagy and apoptosis have not been accurately elucidated. Here, we studied the mechanism of apoptosis and autophagy induced by apoptin and the interaction between autophagy and apoptosis.

Methods: Through crystal violet staining and CCK-8 assay, we analyzed the effect of apoptin in inhibiting liver cancer in vitro, and also analyzed the effect of inhibiting liver cancer in vivo by establishing a nude mouse tumor model. Flow cytometry and fluorescence staining were used to analyze the main types of apoptosis and autophagy induced by apoptin. Subsequently, the relationship between apoptosis and autophagy induced by apoptin was analyzed. Then, flow cytometry was used to analyze the effect of ROS on apoptosis and autophagy mediated by apoptin. Then, the affect of ROS on apoptosis and autophagy mediated by apoptin was analyzed. Finally, the key genes leading to autophagy were analyzed by silencing different genes.

Results: The results showed that apoptin can significantly increase the apoptosis and autophagy of liver cancer cells, and apoptin can cause mitophagy through the increase of NIX protein. Apoptin can also significantly reduce the level of cellular ROS, which is related to the autophagy and apoptosis of liver cancer cells caused by apoptin. The change of ROS may be a key factor causing apoptosis and autophagy.

Conclusion: The above results indicate that the increase of ROS level after apoptin treatment of liver cancer cells leads to the loss of mitochondrial transmembrane potential, which leads to endogenous apoptosis and mitophagy while recruiting NIX. Therefore, ROS may be a key factor connecting endogenous apoptosis and mitophagy induced by apoptin in liver cancer cells.

\section{Background}

Liver cancer is the third largest cause of cancer-related death in the world. In February 2020, the International Agency for Research on Cancer (IARC) of the World Health Organization reported that the number of newly diagnosed cancer cases in the world in 2020 was 19.3 million, which continued to increase from the 18.1 million in 2018; Among them, them, the number of cancer deaths is also stands at the million mark, which is expected to reach 10 million [1]. In 2020, there were 19.3 million new cancer cases in the world, including 4.57 million new cancers in China, accounting for $23.7 \%$ of the global total. As China is the most populous country in the world, the number of new cancers cases far exceeds that of other countries in the world. At present, surgical resection is still one of the most effective methods to treat this disease. However, the current traditional treatment methods are still unsatisfactory.

With the development of molecular biology, cell biology and virology, gene therapy has become a new cancer treatment. Oncolytic virus has shown great advantages, playing the dual functions of viral therapy and gene therapy, and is expected to become an effective method for the treatment of HCC [2]. The 
chicken anemia virus (CAV) genome contains three partially overlapping open reading frames, producing a polycistronic non-spliced RNA that encodes three different proteins. Among them, VP3 is a functional protein of CAV, which is related to virus virulence, can induce apoptosis of target cells, and is a pathogenic protein. The VP3 gene is $366 \mathrm{bp}$ in length, encoding Apoptin protein. The activity of Apoptin depends on phosphorylation and relocation in the nucleus of tumor cells, which does not exist in normal cells[3]. The selective apoptosis effect of Apoptin itself is neither dependent on p53 protein-mediated nor inhibited by the overexpression of $\mathrm{Bcl}-2$, so it is regarded as a new type of anti-tumor biological protein, which is expected to solve the process of tumor gene therapy [4-7].

Cell death is a complex process that is carefully regulated. Apoptosis is the first recognized programmed cell death (PCD) result, and its role and regulatory network have gradually become clear. However, apoptosis is not the only thing that determines the fate of cell death. Autophagy has been proved to regulate cell death along with apoptosis. In some cases, autophagy inhibits apoptosis, but autophagy itself can also induce cell death, or act together with apoptosis, and serve as a standby mechanism to induce cell death in the case of apoptosis defects.

Macroautophagy (the autophagy in this topic specifically refers to macroautophagy) is a high-capacity process, which originated from the target protein of membrane rotation, formed autophagy corpuscles, and then formed autophagy lysosomes, and degraded unnecessary internal substances and other components. This process is composed of more than 30 kinds of proteins and regulatory factors $[8,9]$. The specific substrate P62 (Sequestosome-1), a specific substrate of autophagy, can mediate selective autophagy, such as mitophagy. P62 and LC3-II degrade together with other autophagy substrate proteins, which can reflect the state of autophagy flux [10].

Selective recruitment of autophagy substrate is a special kind of macroautophagy. Mitochondria are usually enclosed by autophagosomes. In some cases, especially under starvation conditions, this phenomenon may be non-specific. However, more and more evidence show that autophagosomes can selectively recognize and encapsulate mitochondria. Studies have shown the connection between mitophagy and Parkinson's disease. The mutation of E3 ubiquitin ligase Parkin and mitochondrial kinase Pink1 will lead to autosomal recessive inheritance of Parkinson's disease. Under the action of Pink1 protein, the Parkin protein in the cytoplasm is translocated to the depolarized mitochondrial surface, which promotes the ubiquitination of mitochondrial proteins, thus inducing mitophagy $[11,12]$. In addition to Parkin, studies have shown that Nix, BNIP3 and FUNDC1 also play an important role in the process of selective mitophagy [13].

In our previous study, a recombinant type 5 adenoviral vector overexpressing Apoptin (Ad-apoptin) was constructed. This delivery system has been proved to be able to express apoptin protein efficiently (and stably) in cells [14]. In fact, ectopic expressions based on transfection reagents has some disadvantages, including (i) lack of experimental consistency and (ii) decrease of expression due to cytototoxic effects and/or inefficiency and instability of the reagent. In previous studies, we conducted a preliminary study 
on apoptosis and autophagy induced by apoptin, and found that apoptin can cause autophagy in tumor cells and interact with apoptosis.

This study intends to use human type 5 adenovirus vector expressing apoptin protein recombinant virus Ad-apoptin to infect liver cancer cells, by detecting whether apoptin has apoptosis and autophagy effects on the cells, and the interaction relationship of apoptosis and autophagy induced by apoptin are studied. It will provide a new theoretical basis for further research on developing tumor drugs based on apoptin in the future.

\section{Materials And Methods}

\section{Human liver cancer cell line, recombinant adenovirus and animals}

The liver cancer cell line HepG-2 and Huh-7 were cryopreserved by the Key laboratory of Jilin Province for Zoonosis Prevention and Control. The two recombinant adenoviruses (Ad-mock and Ad-Apoptin) were constructed and preserved in our laboratory [14].

Female BALB / c nude mice (4-5 weeks old) were purchased from Beijing vital River Laboratory Animal Technology Co., Ltd., and the animal experiment protocols was approved by the Institutional Animal Care and Use Committee (IACUC) of the Changchun University of Chinese Medicine (Approval No. 2020119). All surgeries were performed under anesthesia. After the experiment, the remaining mice were euthanized. The method of euthanasia is intraperitoneal injection of three times the anesthetic dose of sodium pentobarbital ( $150 \mathrm{mg} / \mathrm{kg}$ ) for 2-3 minutes. The euthanasia method followed the the AVMA Guidelines for the Euthanasia of Animals.

\section{Crystal violet staining}

Seed the cells to a 12-well plate and culture for $24 \mathrm{~h}$. Then the recombinant adenovirus infection is performed. After12, 24 and $48 \mathrm{~h}$, take out the 12-well plate, discard the cell supernatant, and wash with PBS three times. Add $600 \mu \mathrm{L} 0.4 \%$ crystal violet staining solution to each well, discard the staining solution after staining for 10 minutes at room temperature, wash with PBS three times, and place in a dry environment, and take photos for analysis.

\section{CCK-8 assay}

The cell suspensions were prepared and added to a 96-well plate. Take out a 96-well cell culture plate at 5 different time points $(6,12,24,48$ and $72 \mathrm{~h})$, and the in each well was discarded and CCK-8 reagent was added, place in a $37^{\circ} \mathrm{C}, 5 \% \mathrm{CO}_{2}$ incubator and incubate for $2 \mathrm{~h}$ in a dark place. After shaking for $10 \mathrm{~s}$, the OD value of the two cells at $450 \mathrm{~nm}$ was measured by microplate reader.

\section{Tumor growth in vivo (xenograft)}


HepG-2 cells $\left(5 \times 10^{6}\right)$ were inoculated into the chest subcutaneously of 5-week-old BALB/c nude mice to establish a tumor model. Then measure the size of the tumor and observe the survival every week. After 42 days of treatment, the animals were euthanized and each tumor was fixed with formalin. Draw the growth curve and calculate the tumor volume:

Tumor volume $\left(\mathrm{mm}^{3}\right)=\left(\right.$ long diameter of tumorxshort diameter of tumor $\left.{ }^{2}\right) / 2$.

Inhibition rate was calculated using the formula:

Tumor inhibition rate $=(1$-treatment group tumor volume/control tumor volume $) \times 100 \%$

\section{Immunochemistry (IHC)}

Tissue sections were deparaffinized, rehydrated, and incubated in $3 \% \mathrm{H}_{2} \mathrm{O}_{2}$ methanol for 15 minutes to eliminate endogenous peroxidase activity. Put the slides in $0.01 \mathrm{M}$ sodium citrate buffer $(\mathrm{pH} 6.0)$ and incubate at $95^{\circ} \mathrm{C}$ for 20 minutes to perform antigen retrieval. Then incubate the slide with the primary antibody overnight at $4^{\circ} \mathrm{C}$. After incubating with the secondary antibody for $1 \mathrm{~h}$ at room temperature, DAB was used for immunostaining, and the sections were counter-stained with hematoxylin. Then analyze the mean density of immunohistochemistry.

\section{Hoechst staining assay}

Seed the cells to a 12-well plate and culture for $24 \mathrm{~h}$. Then the recombinant adenovirus infection is performed. After12, 24 and $48 \mathrm{~h}$, cells were collected, and then $100 \mu \mathrm{L}$ Hoechst dye solution was added. Cells were stained for 15 minutes in the dark, then take $10 \mu \mathrm{l}$ of the stained cell mixture and analyze it through a fluorescence microscope.

\section{Annexin V-FITC/PI staining assay}

Seed the cells to a 12-well plate and culture for $24 \mathrm{~h}$. Then the recombinant adenovirus infection is performed. Then the cells were treated with FITC-coupled Annexin-V apoptotic kit for $12 \mathrm{~h}, 24 \mathrm{~h}$, and $48 \mathrm{~h}$, and the apoptosis was detected by FACS flow cytometry.

\section{Detection of mitochondrial membrane potential}

Seed the cells to a 12-well plate and culture for $24 \mathrm{~h}$. Then the recombinant adenovirus infection is performed. After 12,24 and $48 \mathrm{~h}$, cells were collected, and then $100 \mu \mathrm{L} \mathrm{JC}-1$ dye solution was added. Cells were stained for 15 minutes in the dark, then take $10 \mu \mathrm{l}$ of the stained cell mixture and analyze it through a fluorescence microscope.

The cells were prepared in 96-well plates respectively. The cells were incubated at $5 \% \mathrm{CO}_{2}$ and $37^{\circ} \mathrm{C}$ for 24 hours. Then add diluted $\mathrm{JC}-1$ solution to each well, and incubate at $37^{\circ} \mathrm{C}$ in the dark for 15 minutes. The results were determined by microplate reader.

\section{Western Blotting}


The whole cell protein extract was prepared with RIPA cell lysate containing protease inhibitors. The same amount of protein samples were separated on a $10 \%$ SDS polyacrylamide gel and transferred to a PVDF membrane. Block the membrane with $5 \%$ skim milk for $1-2 \mathrm{~h}$, then incubate the primary antibody overnight at $4^{\circ} \mathrm{C}$, incubate the secondary antibody at room temperature for $2 \mathrm{~h}$, and finally use enzymelinked chemiluminescence (ECL) detection. The results were quantitatively analyzed with chemiluminescence and fluorescence imaging systems. The detailed steps was performed as described previously [15].

\section{Immunofluorescence assay}

Seed the cells to a 12-well plate (with sterile cell slides) and culture for $24 \mathrm{~h}$. Then add Ad-apoptin and different reagents according to different groups. After $48 \mathrm{~h}$, take out the 12-well plate, fix it with $4 \%$ paraformaldehyde for 15 minutes, infiltrate with $0.5 \%$ Triton X-100, block with $1 \%$ bovine serum albumin (BSA) for $2 \mathrm{~h}$, incubate the primary antibody overnight at $4^{\circ} \mathrm{C}$, and wash 3 times with PBS. Secondary antibodies labeled with FITC or $\mathrm{CY} 3$ were incubated for $2 \mathrm{~h}$ and analyzed by Zeiss confocal microscope.

\section{LC3 detection assay}

The cells were transfected with pEGFP-LC3 for 24 hours and then infected with recombinant adenovirus. After 12, 24, and $48 \mathrm{~h}$, the cells were fixed and observed with a fluorescence microscope.

\section{Lysosomal staining assay}

Seed the cells to a 12-well plate and culture for $24 \mathrm{~h}$. Then the recombinant adenovirus infection is performed. After 12, 24 and 48 h, cells were collected, and then $100 \mu \mathrm{L}$ LTR (Lyso-Tracker Red) dye solution was added. Cells were stained for 15 minutes in the dark, then take $10 \mu \mathrm{l}$ of the stained cell mixture and analyze it through a fluorescence microscope.

\section{TMRM staining assay}

Seed the cells to a 12-well plate and culture for $24 \mathrm{~h}$. Then the recombinant adenovirus infection is performed. After 12, 24 and $48 \mathrm{~h}$, cells were collected, and then $100 \mu \mathrm{L}$ TMRM and hoechst dye solution were added. Cells were stained for 15 minutes in the dark, then take $10 \mu \mathrm{l}$ of the stained cell mixture and analyze it through a fluorescence microscope.

\section{Observation on colocalization of lysosomes and mitochondria}

Seed the cells to a 12-well plate and culture for $24 \mathrm{~h}$. Then the recombinant adenovirus infection is performed. After 12, 24 and $48 \mathrm{~h}$, cells were collected, and then $100 \mu \mathrm{L}$ MTG (Mito-Tracker Green) and LTR dye solution were added. Cells were stained for 15 minutes in the dark, then take $10 \mu \mathrm{l}$ of the stained cell mixture and analyze it through a fluorescence microscope.

\section{ROS detection}


Seed the cells to a 12-well plate and culture for $24 \mathrm{~h}$. Then the recombinant adenovirus infection is performed. After 12, 24 and $48 \mathrm{~h}$, cells were collected, and then $100 \mu \mathrm{L}$ DHR dye solution were added. Cells were stained for 30 minutes in the dark. Then flow cytometry was performed to observe the changes in ROS levels.

\section{Statistical analysis}

The statistical analyses were conducted using data from at least three independent experiments, and graph Pad Prism 8 was used to process the data. The data between the two groups were analyzed by random t-test. There was significant difference when $\mathrm{p}<0.05$.

\section{Results}

\section{Inhibitory growth effect of apoptin in liver cancer cells}

Through crystal violet staining and CCK-8 detection, we analyzed the inhibitory effect of apoptin on liver cancer cells (Fig. 1A-D). It was found that apoptin can produce significant cytotoxic effects on liver cancer cells. $(P<0.05)$, and it has a dose-effect relationship and a time-effect relationship. From the CCK-8 experiment, it was found that the inhibition rate reached its peak at $48 \mathrm{~h}$, and the inhibition rate at $72 \mathrm{~h}$ was basically the same as that at $48 \mathrm{~h}(45.02 \%$ and $42.85 \%$, respectively), with no significant difference. Then we also found that the inhibitory effect of Ad-apoptin at $100 \mathrm{MOI}$ was significantly higher than 10 MOI. Obviously, the inhibitory effect is related to dose and time.

\section{The anti-tumor effect of apoptin in vivo}

We established a subcutaneous tumor-bearing model of HepG-2 cells in nude mice. The results showed that compared with the control group, the tumor volume of the Ad-mock group had no obvious change, but the tumor volume was significantly reduced after the injection of Ad-apoptin, which was significantly different from that of the control group $(P<0.05)$ (Fig. 1E-G). In the results of Ki67, it was also found that the proliferation ability of tumor decreased significantly after injection of Ad-apoptin. We also found that injection of Ad-apoptin can prolong the survival time of mice and increase the survival rate (Fig. $1 \mathrm{H})$. The above results are basically consistent with the results obtained in vitro. The results indicated that apoptin had a significant inhibitory effect in vivo and in vitro, and can significantly improve the survival rate of mice.

\section{Apoptin induces apoptosis through the mitochondrial pathway}

In order to analyze whether apoptin kills liver cancer cells through the apoptotic pathway, we performed Hoechst staining and detected the level of apoptosis (Fig. 2A-D). Hoechst staining showed that apoptin cells began to die at $12 \mathrm{~h}$, and large-scale nuclear fragmentation and over-staining were observed at 24 and $48 \mathrm{~h}$, but there was no apoptosis in ad-mock and the control group. From the detection results of 
apoptosis level, it can be seen that the apoptosis level increased from $12 \mathrm{~h}$ and reached its peak at $24 \mathrm{~h}$ (32.13\% for HepG-2 and $26.74 \%$ for Huh-7 cells, respectively). After adding the caspase inhibitor QVD, the apoptosis induced by apoptin and the inhibition rate of liver cancer cells decreased significantly (Fig. 2EF). The immunohistochemical results also found that apoptin can significantly increase the expression level of caspase-3 (Fig. 2G).

In order to determine whether apoptin kills liver cancer cells through the mitochondrial apoptotic pathway, we first performed the $\mathrm{JC}-1$ staining test (Figure $2 \mathrm{H}-\mathrm{K}$ ). After treating the cells with apoptin, the mitochondrial membrane potential gradually decreased. With the passage of time, the number of apoptotic cells gradually increases while the cells are largely depolarized. The mitochondrial membrane potential of the cells treated with both Ad-apoptin and QVD was significantly increased, and the ratio of red fluorescence/green fluorescence was significantly higher than that of the Ad-apoptin group. The above results indicate that apoptin may induce $\mathrm{HCC}$ cell apoptosis by activating the mitochondrial apoptotic pathway.

\section{Changes in autophagy activity}

Next, we analyzed whether apoptin affects the level of autophagy after treating liver cancer cells. As shown in Figures 3A-B and D-E, the cells in the Ad-apoptin group showed obvious LC3 green fluorescence at $12 \mathrm{~h}$, unchanged at $24 \mathrm{~h}$, and increased significantly at $48 \mathrm{~h}$. Similarly, in lysosomal staining, the results were similar to those of LC3. The red fluorescence increased at $12 \mathrm{~h}$, remained unchanged at $24 \mathrm{~h}$, and reached the highest level at $48 \mathrm{~h}$, which indicated that apoptin can inhibit the growth of hepatoma cells and also affect autophagy.

Subsequently, the levels of autophagy-related proteins were analyzed (Fig. 3C and F-G). After adding Adapoptin, the level of LC3 showed a trend of first increasing, then decreasing, and then increasing again, while the trend of P62 was opposite to that of LC3. The above results all indicate that apoptin can affect the level of autophagy in liver cancer cells.

In addition, we analyzed whether the increase in autophagy affects the killing of liver cancer cells by apoptin. The results showed that the inhibition of autophagy can significantly improve the killing effect of apoptin on liver cancer cells, and the inhibition rate can reach $62.83 \%$ at $48 \mathrm{~h}(\mathrm{Fig}$. $3 \mathrm{H})$.

\section{The effect of apoptosis on autophagy}

In order to analyze whether apoptin-induced apoptosis has an effect on autophagy, liver cancer cells were first stained by LTR (Fig. 4A). After adding QVD, the number of lysosomes was found to be significantly reduced, indicating that inhibition of apoptosis may significantly reduce the level of autophagy.

Subsequently, autophagy-related proteins were detected (Fig. 4B-E). After inhibiting apoptosis, we found that the expression levels of autophagy-related proteins LC3-II and Beclin-1 were significantly reduced, while the expression level of $P 62$ was significantly increased $(P<0.05)$. The above results indicate that 
reducing apoptosis can reduce the increase in autophagy caused by apoptin, and the higher the level of apoptosis, the greater the impact.

\section{The effect of autophagy on apoptosis}

Next, in order to analyze whether autophagy has an effect on apoptosis induced by apoptin, we first detected the level of apoptosis (Fig. 4F and K). After inhibiting autophagy, we found that apoptin-induced apoptosis was significantly increased, exceeding the level of apoptosis in the apoptin group alone $(P<0.05)$. The highest level was reached at $48 \mathrm{~h}$, and the apoptotic rate was $63.54 \%$. The above results indicate that inhibiting autophagy can significantly reduce apoptosis of liver cancer cells mediated by apoptin.

Then, the apoptosis-related proteins were detected. After adding 3-MA, it was found that the expression levels of PARP and Caspase-3 increased significantly (Fig. 4G-K). Starting from $12 \mathrm{~h}$, the level of apoptosis-related protein in the 3-MA+Ad-apoptin group was always higher than that in the Ad-apoptin group $(P<0.05)$. The above results once again show that inhibiting autophagy can significantly increase the level of apoptin-mediated apoptosis.

\section{Apoptin can cause mitochondrial autophagy}

TMRM staining was used to further analyze the effect of autophagy on mitochondria (Fig. 5A-C). At $12 \mathrm{~h}$, inhibiting autophagy can significantly reduce the level of mitochondrial membrane potential, and at the same time aggravate mitochondrial damage $(P<0.05)$ (Fig. 5D-E). This indicates that the level of mitochondrial membrane potential is related to autophagy induced by apoptin.

Subsequently, MTG and LTR staining were used to further analyze the relationship between mitochondria and autophagy. (Fig. 5F). After $12 \mathrm{~h}$ h, the green fluorescence of mitochondria decreased and the red fluorescence of lysosome increased. At $24 \mathrm{~h}$, the red fluorescence of lysosome decreased and the green fluorescence continued to decrease. After adding Ad-apoptin, we found that the green fluorescence of mitochondria decreased significantly, while the red fluorescence of lysosomes increased significantly. After inhibiting autophagy, the red and green fluorescence decrease at the same time, and the green fluorescence decreases more rapidly. Interestingly, the areas of increased red fluorescence and decreased green fluorescence of cells in the Ad-apoptin group were at the same position.

Subsequently, we observed by electron microscope that there were a large number of autophagosomes in the Ad-apoptin group, and there were damaged mitochondria in the autophagosomes (Fig. 5G). Then, we analyzed the localization of mitochondrial surface protein and LC3, and found that there was a large number of co-localization in the Ad-apoptin group, and after adding 3-MA, the co-localization phenomenon decreased significantly (Fig. $5 \mathrm{H}$ ). The above results indicate that apoptin may induce the occurrence of mitophagy when inhibiting the proliferation of liver cancer cells.

\section{ROS regulates the cytotoxic effect of apoptin on liver cancer cells}


In order to analyze whether apoptin affects the level of ROS when inhibiting the proliferation of liver cancer cells, we analyzed the level of ROS (Fig. 6A-B). Starting at 12h, the ROS level in the Ad-apoptin group gradually increased and reached a peak at $24 \mathrm{~h}$. After inhibiting apoptosis, it was found that the level of ROS induced by apoptin was reduced to varying degrees. After inhibiting autophagy, it was found that the level of ROS was significantly improved $(P<0.05)$. The above results indicate that apoptin can cause a significant increase in ROS levels, and this is closely related to apoptosis and autophagy.

Subsequently, through CCK-8 assay, we found that after inhibiting the level of ROS, the inhibitory effect of apoptin on liver cancer cells was significantly reduced (Figure 6C). This result is similar to the result after inhibiting apoptosis. This result indicates that apoptin may induce the death of liver cancer cells by increasing the level of ROS.

\section{ROS regulates apoptosis and autophagy changes}

Subsequently, in order to analyze whether ROS will affect apoptosis and autophagy, we conducted qualitative and quantitative analysis of autophagy and apoptosis. First, we found that inhibiting ros can reduce the level of apoptin-mediated apoptosis (Fig. 6D-E). Then the changes in the levels of apoptosisrelated proteins were analyzed (Fig. 6F-I). From the level of apoptosis-related proteins, we can also find that after inhibiting ROS, the expression levels of cleaved-PARP and cleaved-Caspase- 3 can be significantly reduced $(P<0.05)$. The above results indicate that ROS can affect the apoptin-mediated apoptosis of liver cancer cells.

Subsequently, we performed staining analysis on lysosomes (Fig. 6J). After inhibiting ROS, we found that the lysosomal red fluorescence induced by apoptin gradually decreased and was significantly weaker than the Ad-apoptin group at $48 \mathrm{~h}$. Then we analyzed the levels of autophagy-related proteins (Fig. 6K-0). After inhibiting ROS, the expression level of LC3-II and Beclin-1 decreased significantly, while the expression level of P62 increased significantly $(P<0.05)$. The above results indicate that ROS can also significantly affect apoptin-mediated autophagy of liver cancer cells.

\subsection{NIX is a key protein that apoptin causes mitophagy on liver cancer cells}

Next, in order to further analyze the key proteins that cause mitophagy, we performed the detection of mitophagy-related proteins. The results showed that the change trend of NIX and BNIP3 was similar to that of autophagy induced by apoptin, and the decrease of NIX was more significant after adding NAC (Fig. 7A). In immunohistochemical experiments, we also found that apoptin can increase the level of apoptosis and autophagy, and increase the expression of NIX and BNIP3; Inhibition of ROS can reduce the level of apoptosis and autophagy, and reduce the expression of NIX and BNIP3, which has a more significant effect on NIX (Fig. 7B). After silencing NIX and BNIP3, we found that the expression of cleaved-PARP and cleaved-caspase-3 increased significantly. On the contrary, the expression of LC3 II and beclin1 decreased significantly (Fig. 7C). Similar results were obtained in immunohistochemical experiments (Fig. 7D). To analyze the role of NIX and BNIP3 in mitophagy, we analyzed the inhibitory 
effect of apoptin on liver cancer cells after NIX and BNIP3 were silenced and autophagy inhibited. The results showed that the addition of 3-MA and NIX silenced had similar results, but there was no significant difference after BNIP3 silencing (Fig. 7E).

We also inhibited autophagy with 3-MA, and analyzed the localization of NIX and mitochondrial membrane proteins (Fig. 7F). The results found that the two proteins in the Ad-apoptin group had a strong co-localization phenomenon, which was significantly reduced after inhibition of autophagy. Finally, we performed immunoprecipitation experiments, and found that there was a significant interaction between NIX and LC3 in the Ad-apoptin group, and the effect of inhibiting autophagy and ROS was significantly reduced (Fig. 7G). All the above results indicate that NIX is a key protein of mitophagy induced by apoptin.

\section{Discussion}

Cancer is one of the most serious diseases in the world, and its morbidity and mortality are rising rapidly in the world, which seriously threaten human health. Among all cancers, the morbidity and mortality of liver cancer is also increasing year by year. Hepatocellular carcinoma (HCC) is a highly malignant cancer, which is characterized by concealment, strong invasion and rapid progress. At present, surgery and chemotherapy are still the main treatments for liver cancer. Although chemotherapy can relieve the condition of patients with liver cancer, most patients will relapse in a short time due to chemotherapy resistance. At present, the mechanism of drug resistance in chemotherapy is not clear, and patients will also have serious toxic reactions during chemotherapy. Although some achievements have been made in the field of precision medicine, such as molecular targeted therapy and immunotherapy, most of these studies are still in the second-line or above clinical stage, which is not only small in sample size, but also inconsistent in research results.

Apoptin, as a kind of protein inducing specific apoptosis of tumor cells, is considered as a new anti-tumor biological agent because it is not inhibited by p53-mediated or Bcl-2 overexpression. Through the study of apoptin, it is also expected to solve the problem of low efficiency, poor specificity and drug resistance in cancer treatment.

In this study, we first analyzed the cytotoxic effect of apoptin on liver cancer cells through a variety of tests in vivo and in vitro. We found that apoptin can significantly cause the death of liver cancer cells, which is similar to the results we have obtained in other tumor cells $[4,7,14,16-18]$. Moreover, we also found that the killing ability of apoptin on liver cancer cells is higher than that on other tumor cells, indicating that apoptin has shown a more potential therapeutic effect in the treatment of liver cancer. Interestingly, it was found in vivo experiments that apoptin can not only inhibit the growth of tumors, but also increase the survival time of animals. Therefore, apoptin can significantly kill liver cancer cells both in vivo and in vitro.

Studies have shown that apoptin mainly kills a variety of tumor cells through the apoptosis mechanism [19]. Including the activation of caspase protein in the human osteosarcoma cell line (Saos-2) to initiate 
apoptotic killer cells [20]; it also kills Jurkat T cells through a death receptor-independent pathway [21]. In this study, we found that apoptin can significantly increase the level of cell apoptosis, and after adding caspase inhibitors, the killing effect of apoptin on liver cancer cells was significantly reduced. This result is similar to the results of other studies, that is, apoptin mainly kills various tumor cells through the apoptosis mechanism [19]. Subsequent analysis of mitochondrial membrane changes potentially revealed that apoptin can significantly damage mitochondria and lead to depolarization of cells. These results show that apoptin can kill liver cancer cells by causing changes in mitochondrial membrane potential, indicating that it may lead to liver cancer cells through endogenous apoptotic pathways.

Autophagy refers to the process of cellular self-digestion marked by the appearance of autophagosomes in the cytoplasm as the proteins and organelles that are damaged, denatured or senescent in cells are transported to lysosomes, and the lysosomes digest and degrade them. The autophagosome, which envelops part of the cytoplasm and organelles with a double-layer membrane structure, is taken as a judgment index. Studies have shown that tumors are closely related to autophagy, and many clinical drugs regulating autophagy play an important role in tumor treatment. Here, we analyzed by staining autophagy-related proteins and lysosomes that apoptin kills HCC cells while affecting autophagy, and that autophagy is a protective autophagy reaction.

Studies have shown that the stimulation of cells by external factors can cause autophagy, and autophagy can also lead to apoptosis [22]. The relationship between these two types of programmed death can be divided into the following categories: (i) Autophagy can alleviate the stress state of the environment on the cell, thereby maintaining the normal function of the cell [23]; (ii) Autophagy can also provide apoptosis. ATP required for death or activation of caspase activity to promote the process of apoptosis [24-27]; (iv) Autophagy can inhibit apoptosis by degrading damaged organelles and maintaining normal cell functions, such as mitochondria (i.e. mitochondrial autophagy) [28-30].

Here, we studied the relationship between apoptosis and autophagy induced by apoptin. We found that inhibiting apoptosis can reduce the level of apoptin-mediated autophagy in liver cancer cells. On the contrary, inhibiting autophagy can increase the level of apoptosis of liver cancer cells mediated by apoptin. In addition, we also analyzed the influence of autophagy on mitochondria, and found that autophagy may have some influence on the changes of matrix metalloproteinases (MMP) induced by apoptin. Then, we analyzed the relationship between mitochondria and lysosomes. We first discovered that mitochondria and lysosomes co-localized. Subsequently, we observed the co-localization of LC3 and HSP60, and found that these two proteins had a strong co-localization phenomenon after treatment with apoptin. Therefore, we preliminarily judged that apoptin may induce mitophagy and apoptosis.

As a very important factor in mitochondrial damage, ROS also played a key role in this study. We found that apoptin can increase the level of ROS in liver cancer cells, and ROS can influence the changes in apoptosis and autophagy. Similarly, apoptosis and autophagy can also have adverse effects on the level of ROS. This indicates that apoptin can influence apoptosis and autophagy by adjusting the level of ROS. 
Next, in order to further understand the key proteins that cause mitophagy, we carried out the detection of mitophagy-related proteins. The results showed that the changing trend of NIX was similar to the changing trend of autophagy in liver cancer cells induced by apoptin, and the change of NIX would significantly affect the level of autophagy. Subsequently, inhibition of ROS was also found to significantly decrease the expression level of NIX. Finally, through immunoprecipitation experiments, it was found that there was a significant interaction between NIX and LC 3 , and the effect of inhibiting autophagy and ROS was significantly reduced. All the above results indicate that NIX is a key protein of mitophagy induced by apoptin.

\section{Conclusions}

In summary, apoptin can significantly kill liver cancer cells, mainly through endogenous apoptosis leading to the death of liver cancer cells, but also has a significant impact on autophagy. Here, when apoptin kills liver cancer cells by inducing apoptosis, autophagy plays a protective role. Through multilevel studies, it is found that the increase of ROS levels leads to the loss of mitochondrial transmembrane potential after the action of apoptin on liver cancer cells, which leads to apoptosis and mitophagy at the same time of recruiting a large number of NIX protein (Fig. 8). Therefore, our research shows that ROS may be a key factor connecting apoptin-induced apoptosis and mitophagy in liver cancer cells.

\section{Abbreviations}

ROS

reactive oxygen species

PCD

programmed cell death

CAV

chicken anemia virus

DRs

death receptors

FADD

Floationg Point Addition

Atg

autophagy-related genes

$\mathrm{MOI}$

multiple of infection

QVD

Q-VD-OPh

DMEM

Dulbecco's Modified Eagle Media

CQ 
Chloroquine

NAC

n-acetyl cysteine

LTR

Lyso-Tracker Red

MTG

Mito-Tracker Green

\section{Declarations}

\section{Acknowledgments}

This work was supported by the Jilin Province Youth Scientific and Technological Talent Support Project (Grant No. QT202111).

\section{Author contributions}

Conceived and designed the experiments: Yiquan Li, Xiao Li, Jinbo Fang and Yilong Zhu. Performed the experiments: Yiquan Li, Yilong Zhu, Wenjie Li, Shanzhi Li, Xing Liu, Zirui Liu, Gaojie Song and Ningyi Jin. Analyzed the data: Yiquan Li, Xiao Li, Lili Sun and Yilong Zhu. Contributed reagents/materials/analysis tools: Yilong Zhu, Chao Shang, Shanzhi Li, Jianan Cong and Bing Bai. Wrote the paper: Yiquan Li and Yilong Zhu. All authors read and approved the final manuscript.

\section{Availability of supporting data}

The datasets used and analyzed during the current study are available from the corresponding author on reasonable request.

\section{Ethics approval and consent to participate}

All applicable international, national, and/or institutional guidelines for the care and use of animals were followed.

\section{Consent for publication}

Not applicable.

\section{Competing interests}


The authors declare that they have no competing interests.

\section{References}

1. Sung H, Ferlay J, Siegel RL, Laversanne M, Soerjomataram I, Jemal A, Bray F. Global Cancer Statistics 2020: GLOBOCAN Estimates of Incidence and Mortality Worldwide for 36 Cancers in 185 Countries. CA Cancer J Clin. 2021;71:209-49.

2. Gu D, Kelly TN, Wu X, Chen J, Samet JM, Huang JF, Zhu M, Chen JC, Chen CS, Duan X, et al. Mortality attributable to smoking in China. N Engl J Med. 2009;360:150-9.

3. Backendorf C, Noteborn MHM: Apoptin towards safe and efficient anticancer therapies. In Advances in Experimental Medicine and Biology, vol. 818. pp. 39-59; 2014:39-59.

4. Liu L, Wu W, Zhu G, Liu L, Guan G, Li X, Jin N, Chi B. Therapeutic efficacy of an hTERT promoterdriven oncolytic adenovirus that expresses apoptin in gastric carcinoma. Int $\mathrm{J}$ Mol Med. 2012;30:747-54.

5. Zhang M, Wang J, Li C, Hu N, Wang K, Ji H, He D, Quan C, Li X, Jin N, Li Y. Potent growth-inhibitory effect of a dual cancer-specific oncolytic adenovirus expressing apoptin on prostate carcinoma. Int $J$ Oncol. 2013;42:1052-60.

6. Qi Y, Guo H, Hu N, He D, Zhang S, Chu Y, Huang Y, Li X, Sun L, Jin N. Preclinical pharmacology and toxicology study of Ad-hTERT-E1a-Apoptin, a novel dual cancer-specific oncolytic adenovirus. Toxicol Appl Pharmacol. 2014;280:362-9.

7. Yang G, Meng X, Sun L, Hu N, Jiang S, Sheng Y, Chen Z, Zhou Y, Chen D, Li X, Jin N. Antitumor effects of a dual cancer-specific oncolytic adenovirus on colorectal cancer in vitro and in vivo. Exp Ther Med. 2015;9:327-34.

8. Levy JMM, Towers CG, Thorburn A. Targeting autophagy in cancer. Nat Rev Cancer. 2017;17:528-42.

9. Mizushima N, Yoshimori T, Ohsumi Y. The role of Atg proteins in autophagosome formation. Annu Rev Cell Dev Biol. 2011;27:107-32.

10. Klionsky DJ, Abdelmohsen K, Abe A, Abedin MJ, Abeliovich H, Acevedo Arozena A, Adachi H, Adams $\mathrm{CM}$, Adams PD, Adeli $\mathrm{K}$, et al: Guidelines for the use and interpretation of assays for monitoring autophagy (3rd edition). Autophagy 2016, 12:1-222.

11. Youle RJ, Narendra DP. Mechanisms of mitophagy. Nat Rev Mol Cell Biol. 2010;12:9-14.

12. Narendra D, Tanaka A, Suen DF, Youle RJ. Parkin is recruited selectively to impaired mitochondria and promotes their autophagy. J Cell Biol. 2008;183:795-803.

13. Tolkovsky AM. Mitophagy. Biochim Biophys Acta. 2009;1793:1508-15.

14. Li X, Liu Y, Wen Z, Li C, Lu H, Tian M, Jin K, Sun L, Gao P, Yang E, et al. Potent anti-tumor effects of a dual specific oncolytic adenovirus expressing apoptin in vitro and in vivo. Mol Cancer. 2010;9:10.

15. Li X, Jin N, Mi Z, Lian H, Sun L, Li X, Zheng H. Antitumor effects of a recombinant fowlpox virus expressing Apoptin in vivo and in vitro. Int J Cancer. 2006;119:2948-57. 
16. Jin J, Zhu Y, Sun F, Chen Z, Chen S, Li Y, Li W, Li M, Cui C, Cui Y, et al. Synergistic antitumor effect of the combination of a dual cancer-specific oncolytic adenovirus and cisplatin on lung cancer cells. Oncol Lett. 2018;16:6275-82.

17. Chen S, Li YQ, Yin XZ, Li SZ, Zhu YL, Fan YY, Li WJ, Cui YL, Zhao J, Li X, et al. Recombinant adenoviruses expressing apoptin suppress the growth of MCF7 breast cancer cells and affect cell autophagy. Oncol Rep. 2019;41:2818-32.

18. Cui CX, Li YQ, Sun YJ, Zhu YL, Fang JB, Bai B, Li WJ, Li SZ, Ma YZ, Li X, et al: Antitumor effect of a dual cancer-specific oncolytic adenovirus on prostate cancer PC-3 cells. Urol Oncol 2019, 37:352 e351-352 e318.

19. Zhang C, Zhou D. Adenoviral vector-based strategies against infectious disease and cancer. Hum Vaccin Immunother. 2016;12:2064-74.

20. Danen-van Oorschot AA, van Der Eb AJ, Noteborn MH. The chicken anemia virus-derived protein apoptin requires activation of caspases for induction of apoptosis in human tumor cells. J Virol. 2000;74:7072-8.

21. Maddika S, Booy EP, Johar D, Gibson SB, Ghavami S, Los M. Cancer-specific toxicity of apoptin is independent of death receptors but involves the loss of mitochondrial membrane potential and the release of mitochondrial cell-death mediators by a Nur77-dependent pathway. J Cell Sci. 2005;118:4485-93.

22. Chaabane W, Appell ML. Interconnections between apoptotic and autophagic pathways during thiopurine-induced toxicity in cancer cells: the role of reactive oxygen species. Oncotarget. 2016;7:75616-34.

23. Adams JM, Cory S. The Bcl-2 apoptotic switch in cancer development and therapy. Oncogene. 2007;26:1324-37.

24. Bovellan M, Fritzsche M, Stevens C, Charras G. Death-associated protein kinase (DAPK) and signal transduction: blebbing in programmed cell death. FEBS J. 2010;277:58-65.

25. Espert L, Denizot M, Grimaldi M, Robert-Hebmann V, Gay B, Varbanov M, Codogno P, BiardPiechaczyk M. Autophagy is involved in T cell death after binding of HIV-1 envelope proteins to CXCR4. J Clin Invest. 2006;116:2161-72.

26. Yu L, Alva A, Su H, Dutt P, Freundt E, Welsh S, Baehrecke EH, Lenardo MJ. Regulation of an ATG7beclin 1 program of autophagic cell death by caspase-8. Science. 2004;304:1500-2.

27. Yu L, Lenardo MJ, Baehrecke EH. Autophagy and caspases: a new cell death program. Cell Cycle. 2004;3:1124-6.

28. Kraft C, Peter M, Hofmann K. Selective autophagy: ubiquitin-mediated recognition and beyond. Nat Cell Biol. 2010;12:836-41.

29. Levine B, Klionsky DJ. Development by self-digestion: molecular mechanisms and biological functions of autophagy. Dev Cell. 2004;6:463-77.

30. Kirkin V, McEwan DG, Novak I, Dikic I. A role for ubiquitin in selective autophagy. Mol Cell. 2009;34:259-69. 


\section{Figures}

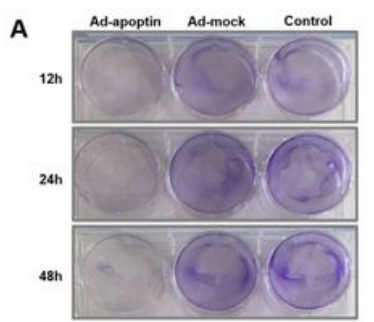

C

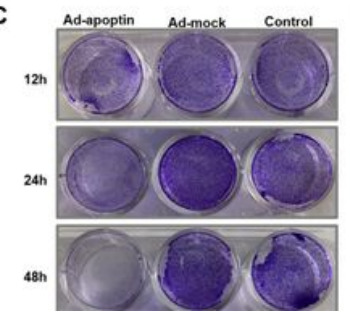

B

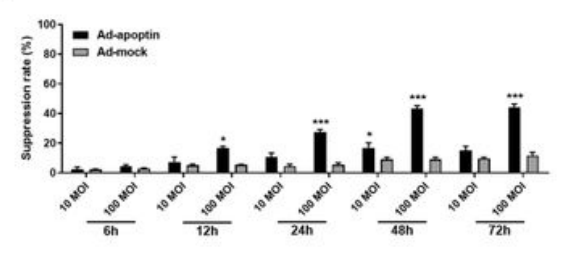

D

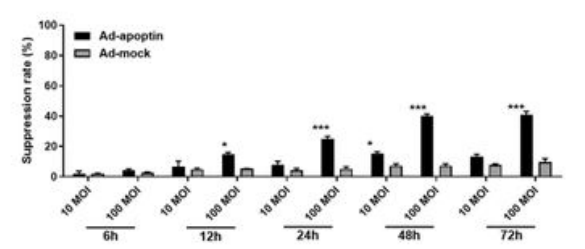

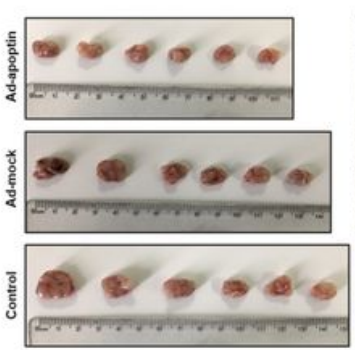

G

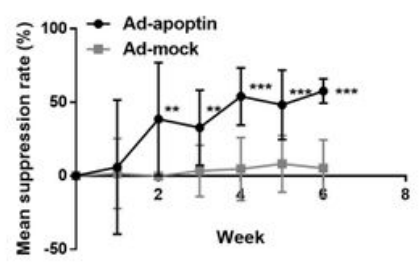

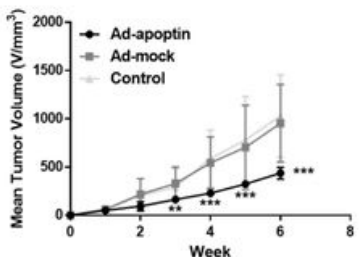

$\mathrm{H}$

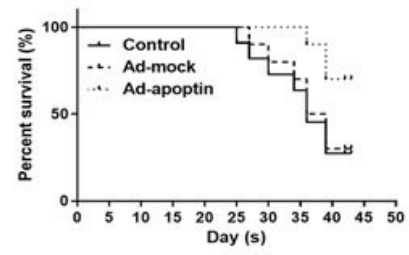

Figure 1

The cytotoxic effect of apoptin on hepatocarcinoma cells.

The crystal violet staining and CCK-8 assay analyzes the inhibition effect of Ad-apoptin on HepG-2 (A-B) and Huh-7 (C-D) cells. (E-G) Length and width of xenograft tumors measured weekly for 6 weeks using Vernier calipers. $(\mathrm{H})$ The survival rate of tumor bearing mice was recorded every day for 5 weeks. Data were representative of three independent experiments $(n=3)$. The unpaired Student's $t$ test was used. *P< $0.05,{ }^{\star *} \mathrm{p}<0.01$. 

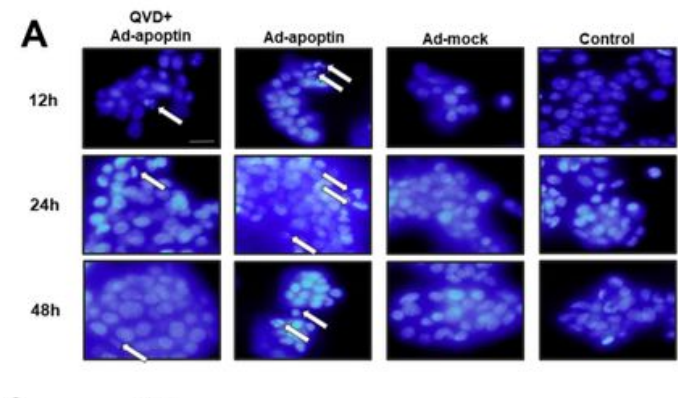

B

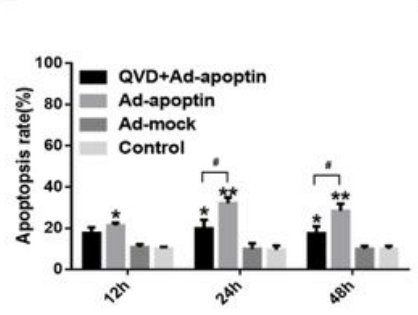

E

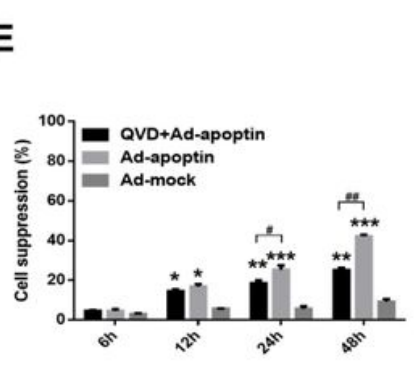

G

Cleaved-Caspase-3
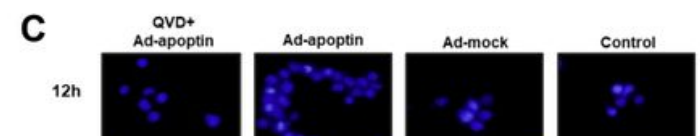

D

$\mathbf{F}$

100 ᄀ

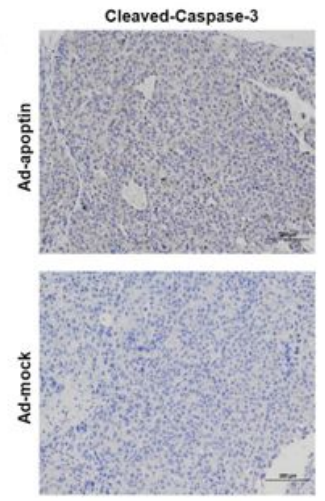

\section{Figure 2}

Pathway of apoptin inducing the death of hepatocarcinoma cells.

The apoptosis of HepG-2 (A-B) and Huh-7 (C-D) cells were detected by Hoechst staining and Annexin VFITC/PI staining. After the addition of the caspase inhibitor QVD $(20 \mu \mathrm{M})$ to the cells infected with Adapoptin, the inhibition rate of HepG-2 (E) and Huh-7 (F) cells were detected by CCK-8 assay. (G) Expression of cleaved caspase- 3 in the xenograft tumours tissues was detected by IHC. JC- 1 staining assays analyzes the changes of mitochondrial membrane potential (MMP) of HepG-2 (H-I) and Huh-7 (JK) cells. The scale bar equals $100 \mu \mathrm{m}$. Data were representative of three independent experiments $(n=3)$. The unpaired Student's test-test was used. ${ }^{\star} p<0.05,{ }^{\star \star} p<0.01,{ }^{\star \star \star} p<0.001 .\left({ }^{\#} p<0.05,{ }^{\# \#} p<0.01\right)$ when compared with Ad-apoptin. 
A
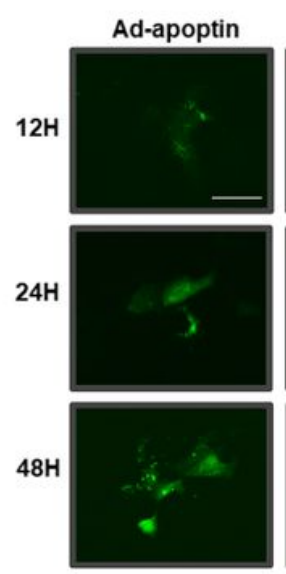

D
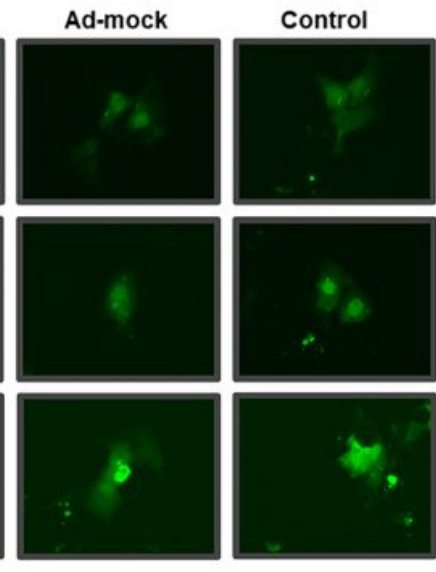

B
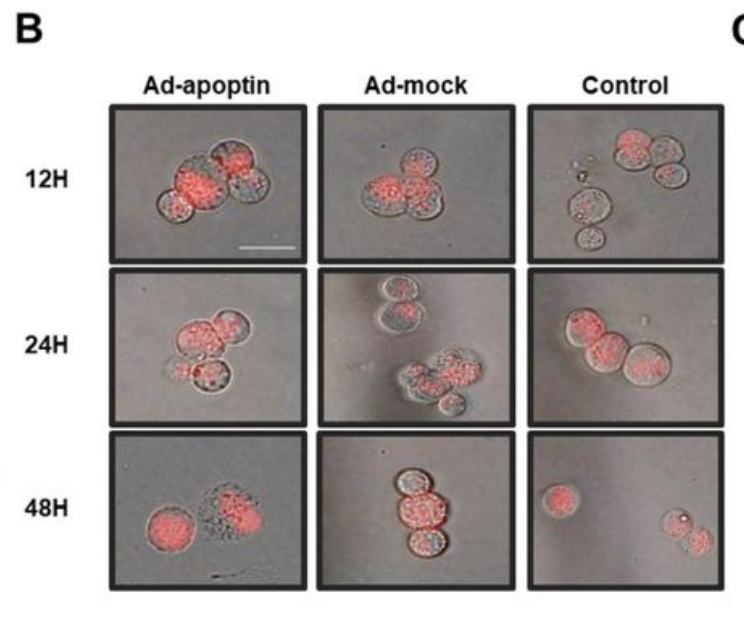

E
C

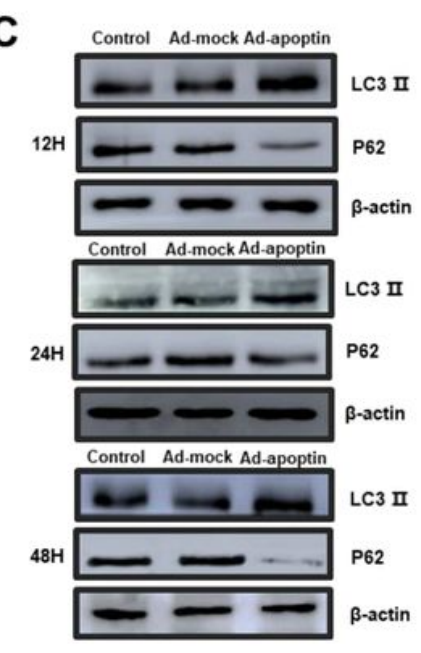

$\mathbf{F}$

\section{Figure 3}

Identification of Effect of autophagy on apoptosis of liver cancer cells by apoptin.

Analyze changes in autophagic flux with LC3-GFP plasmid in HepG-2 (A) and Huh-7 (D) cells. After LTR staining in HepG-2 (B) and Huh-7 (E) cells, the changes in lysosomal fluorescence intensity were observed by fluorescence microscope. Western blot assay analyzes autophagy and apoptosis-related protein levels in HepG-2 (C) and Huh-7 (F) cells. (G) Expression of cleaved LC3 in the xenograft tumours tissues was detected by IHC. (H) After the addition of the autophagy inhibitor 3-MA (2 mM) to the cells infected with Ad-apoptin, the inhibition rate of HepG-2 and Huh-7 cells were detected by CCK-8 assay. The scale bar 
equals $50 \mu \mathrm{m}$. Data were representative of three independent experiments $(n=3)$. The unpaired Student's test-test was used. ${ }^{\star} p<0.05,{ }^{\star \star} p<0.01,{ }^{* \star *} p<0.001$. $\left({ }^{\#} p<0.05,{ }^{\# \#} p<0.01\right)$ when compared with Ad-apoptin.

A
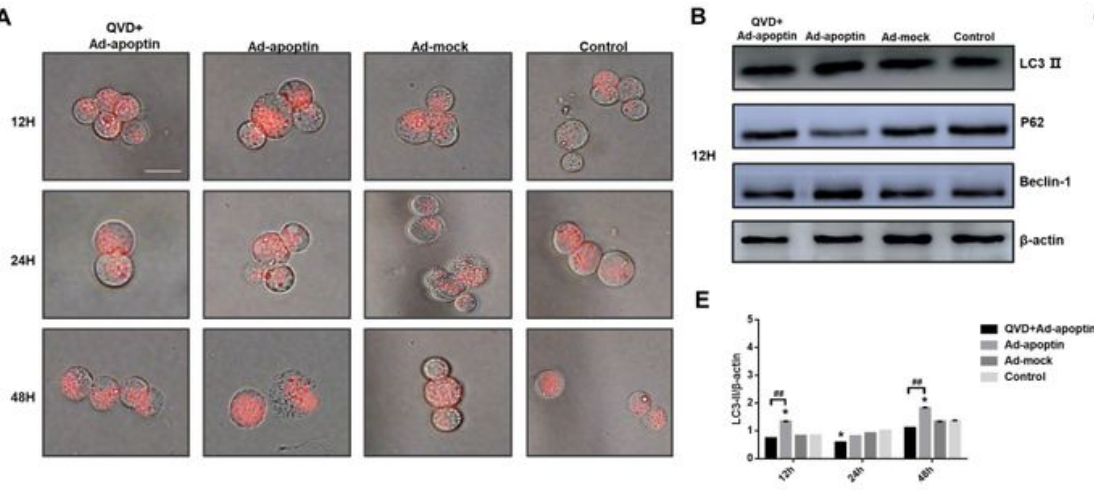

C
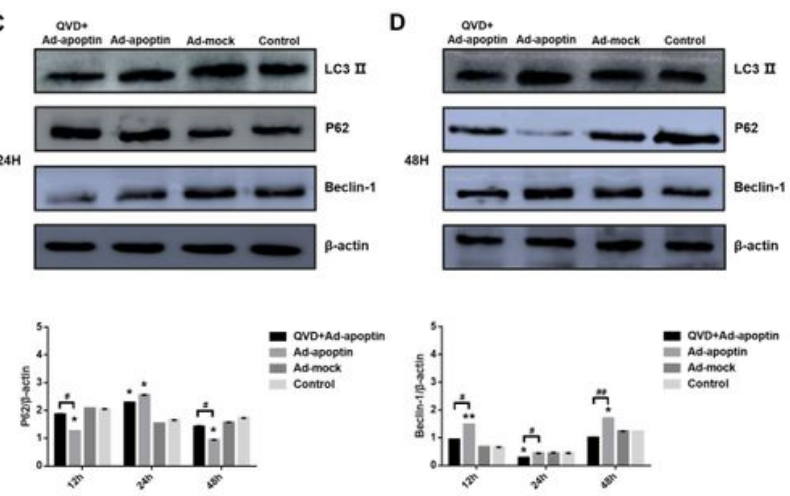

F
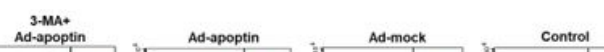

G
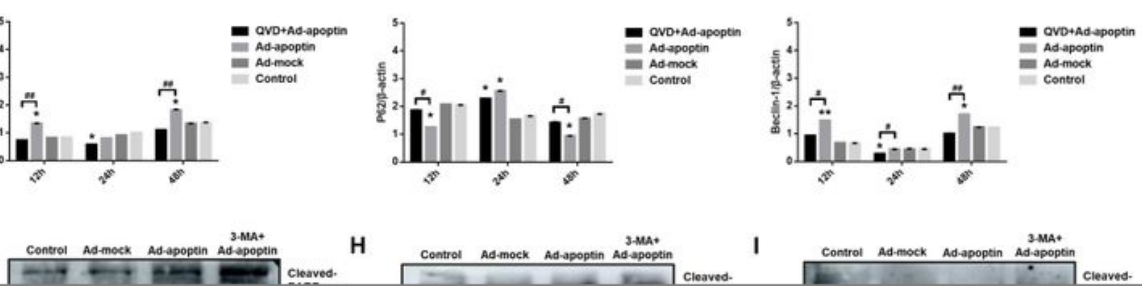

\section{Figure 4}

\section{Analysis of the relationship between apoptin-mediated autophagy and apoptosis.}

(A) After LTR staining in HepG-2 cells, the changes in lysosomal fluorescence intensity were observed by fluorescence microscope. (B-E) Western blot analysis of autophagy-related protein expression. (F) and (J) Apoptosis analysis by flow cytometry after Annexin-V FITC/PI staining. (G-I) and (K) Western blot analysis of apoptosis-related protein expression. The scale bar equals $50 \mu \mathrm{m}$. Data were representative of three independent experiments $(\mathrm{n}=3)$. The unpaired Student's test-test was used. ${ }^{\star} p<0.05,{ }^{\star \star} p<0.01$, $\star \star \star p<0.001$. $\left(\# p<0.05,{ }^{\# \#} p<0.01\right)$ when compared with Ad-apoptin.

\section{Figure 5}

Analysis of the relationship between apoptin-mediated autophagy and mitochondria.

(A-C) Qualitative analysis of changes in mitochondrial membrane potential by TMRM staining. (D-E) Quantitative analysis of changes in mitochondrial membrane potential by TMRM staining. (F) Analyze the co-localization of mitochondria and lysosomes by MTG and LTR staining. (G) Observe the formation of autophagosomes and the types of contents under the electron microscope. $(\mathrm{H})$ Analyze the colocalization of LC3 and HSP60 by immunofluorescence staining. The scale bar equals $50 \mu \mathrm{m}$. Data were 
representative of three independent experiments $(\mathrm{n}=3)$. The unpaired Student's test-test was used. * $p$ $<0.05,{ }^{\star \star} p<0.01,{ }^{\star \star \star} p<0.001$. ( $\left.{ }^{\#} p<0.05,{ }^{\# \#} p<0.01\right)$ when compared with Ad-apoptin.

\section{Figure 6}

\section{The role of ROS in apoptin-mediated autophagy and apoptosis.}

(A-B) Quantitative analysis of changes in mitochondrial membrane potential by DHR staining. (C) After the addition of the ROS inhibitor NAC $(10 \mathrm{mM})$ to the cells infected with Ad-apoptin, the inhibition rate of HepG-2 cells were detected by CCK-8 assay. (D-E) Quantitative analysis of changes in apoptosis level by Annexin-V FITC/PI assay. (F-I) Western blot analysis of apoptosis-related protein expression. (J) After LTR staining, the changes in lysosomal fluorescence intensity were observed by fluorescence microscope. (K0) Western blot analysis of autophagy-related protein expression. The scale bar equals $50 \mu \mathrm{m}$. Data were representative of three independent experiments $(n=3)$. The unpaired Student's test-test was used. * $p$ $<0.05,{ }^{\star \star} p<0.01,{ }^{\star \star \star} p<0.001$. ( $\left.{ }^{\#} p<0.05,{ }^{\# \#} p<0.01\right)$ when compared with Ad-apoptin.

\section{Figure 7}

\section{Effect of NIX protein on apoptin-induced mitotophagy in liver cancer cells}

(A) After the addition of the ROS inhibitor NAC to the cells infected with Ad-apoptin, the mitophagy related protein level was detected by Western blot assay. (B) Expression of LC3, cleaved caspase-3, NIX, BNIP3 and Ki67 in the xenograft tumours tissues was detected by IHC. (C) Western blot assay analyzes the changes of apoptosis and autophagy related proteins level in MCF-7/ADR cells after silencing NIX and BNIP3. (D) Expression of LC3and cleaved caspase-3 in the xenograft tumours tissues was detected by IHC after silencing NIX and BNIP3. (E) After silencing NIX and BNIP3, the inhibition rate of HepG-2 cells were detected by CCK-8 assay. (F) Analyze the co-localization of NIX and HSP60 by immunofluorescence staining. (G) Co-immunoprecipitation assay to analyze the interaction between LC3 and NIX. The scale bar equals $20 \mu \mathrm{m}$. Data are shown as mean \pm SD $\left({ }^{\star} p<0.05\right.$, $\left.{ }^{\star \star} p<0.01,{ }^{\star \star \star} p<0.001\right)$ when compared with controls. 

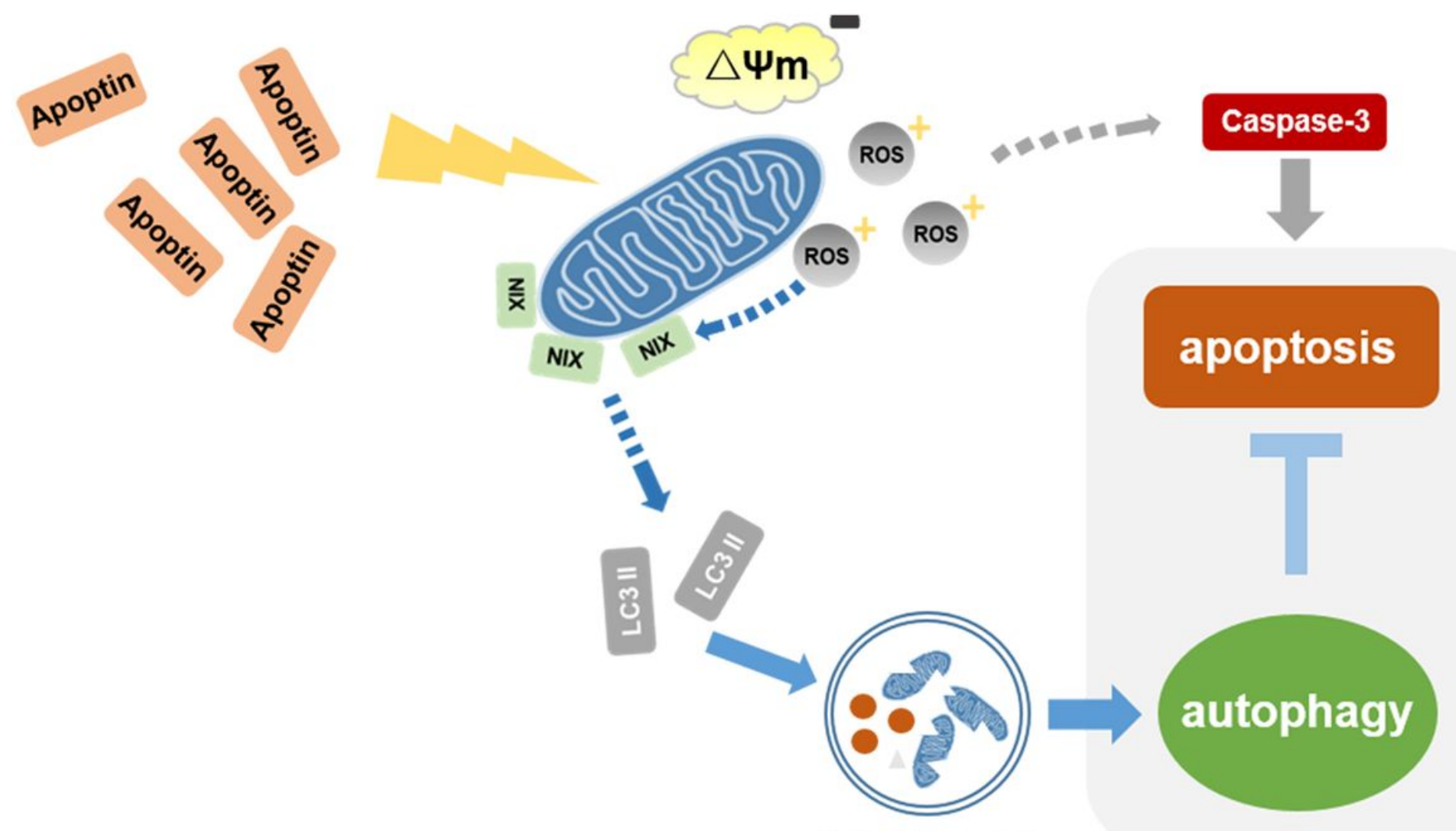

Autophagosome

Figure 8

Schematic representation of the proposed mechanism demonstrating the the correlation between apoptosis and autophagy induced by apoptin.

Apoptin promotes the production of ROS, and induces apoptosis by promoting the lysis of caspase-3, and at the same time increases the NIX protein to cause the occurrence of mitophagy. Autophagy produced in this process is a protective mechanism that inhibits cell apoptosis. 